\title{
Assessment of subclinical cardiovascular autonomic neuropathy among type II diabetic patients: a study from Karachi, Pakistan
}

\begin{abstract}
Introduction: Diabetes Mellitus (DM) is one of the most significant killer diseases worldwide. Cardiovascular Autonomic Neuropathy (CAN) is among the severest but least understood complication of diabetes which remains undiagnosed for several years because of its asymptomatic nature. CAN affect the quality of life very badly and raises the risk of mortality as it is associated with dysfunction of cardiac system. Early diagnosis of CAN is needed to prevent high morbidity and mortality rates.
\end{abstract}

Aim: To identify the presence of Cardiac autonomic neuropathy in type II diabetic patients.

Methods: Seventy two cases of diabetes mellitus (type 2) with no clinical evidence of cardiac disease were subjected to cardiac autonomic function tests according to Ewing's criteria, included the heart rate variability during deep breathing, Valsalva maneuver ratio, heart rate response on standing and BP response to standing and $\mathrm{BP}$ response to sustained handgrip to find the prevalence of CAN. Patients were categorized as with no CAN, early, definite and severe type of CAN depending upon abnormality of one or more tests.

Results: In this study prevalence of CAN was $40 \%$ out of which early, definite and severe involvement was found in $13.9 . \%, 12.5 \%$ and $13.9 \%$ respectively.

Cardiac autonomic function tests (CARTs) of heart rate variability during deep breathing, Valsalva maneuver ratio, heart rate response on standing and BP response to standing and BP response to sustained handgrip found abnormal response in $36.1 \%$, $21 \%, 30.5 \%, 8.4 \%$ and $26.4 \%$ respectively. Tachycardia. Orthostatic hypotension, numbness and constipation were significantly associated with CAN

Conclusion: Prevalence of CAN among diabetics was $40 \%$ and parasympathetic cardiac autonomic function tests are more sensitive for the detection of CAN than sympathetic cardiac autonomic function tests. Development of CAN in diabetic patients lead to poor prognosis, increased silent myocardial infarction and sudden cardiac death hence all diabetic patients should be routinely evaluated for CAN using these feasible bedside tests
Volume 6 Issue I - 2019

\author{
Tallat N, ${ }^{1,4}$ Savanur A, ${ }^{2}$ Fatima A,' Noorunnisa \\ M,' Fizza A, ${ }^{3}$ Khalid $M,{ }^{3,4}$ Qureshi $M A^{4}$ \\ 'Sindh Medical College, Jinnah Sindh Medical University, Pakistan \\ ${ }^{2}$ Department of Physiology, University of Karachi, Pakistan \\ ${ }^{3}$ Civil Hospital, Karachi, Pakistan \\ ${ }^{4}$ Dow University of Health sciences, Pakistan
}

Correspondence: Tallat Naz, Physiology Instructor, Department of Physiology, Sindh Medical College, Jinnah Sindh Medical University, Karachi, Pakistan, Email tallatnaz2002@yahoo.com

Received: December 30, 2018 | Published: February 07, 2019

\section{Introduction}

Diabetes mellitus (DM) is a collection of metabolic disorders unified by presence of hyperglycemia. This rapidly escalating healthcare challenging disease causes economic, social and healthcare burden due to its high prevalence and secondary patho-physiological changes in several systems of the body. Being a global epidemic, DM is estimated to affect 424.9 million people globally. Pakistan is among top 10 countries with disease burden of approximately $8.3 \%$ (7.5 million).

Many complications follow the onset of diabetes. Major diabetic complications are classified into macrovascular and microvascular. Diabetic cardiovascular autonomic neuropathy is one of the microvascular complications and confer high morbidity and mortality to diabetic patients. Several mechanisms were postulated for diabetic neuropathy e.g. high activity of polyol 'pathway and protein kinase (PKc), advance-glycation-end-products (AGEs), ${ }^{2}$ excessive oxidative stress and formation of reactive oxygen species (ROS).

CAN is a serious chronic complication of diabetes and an independent predictor of cardiovascular disease mortality. It causes abnormalities in heart rate as well as blood pressure control. It is very difficult to detect Diabetic Cardiovascular Autonomic Neuropathy (DCAN) at early stages because it stays silent for several years, that is why it is often over looked during both in diagnosis and treatment of diabetes. It is important to improve our understanding regarding CAN and its effects on cardiovascular system so as to incorporate latest treatment and reduce the disease burden of DCAN in diabetes patients.

It is also, recommended by several professional bodies, ${ }^{4-6}$ to perform subclinical assessment of CAN by utilizing CARTs as soon as T2DM is diagnosed. Early detection has significant role in quantifying the disease burden and planning health policies to tackle this worldwide problem.

Ewing's CARTs are considered as Gold standard ${ }^{6}$ in CAN therefore, consistently been used for its subclinical assessment. ${ }^{7,8}$ These tests interrupt the autonomic nervous system (ANS) around its operating point at rest, by deep breathing, changing posture and by performing the Valsalva maneuver to study heart rate changes, whereas orthostatic hypotension and sustained hand grip to study BP changes and responses were observed. ${ }^{6}$ 
Being the biggest city and central hub of trade, Karachi has a large population which shown to have high prevalence rate of DM $(9.4 \%)^{9}$ and its underlying complications. Despite of high prevalence, still Karachi is lacking behind regarding any study conducted on this devastating complication. Hence, this study was conducted with the aim to find the prevalence of DCAN in population of Karachi Pakistan.

\section{Materials and methods}

This cross-sectional study was conducted at Department of Medicine, Civil Hospital Karachi, Pakistan. Seventy-two clinically diagnosed type II Diabetic patients with at least three years duration of diabetes, from Diabetic clinic were selected for this study by purposive sampling technique. All patients were subjected to detailed history and systemic examination. Patients with history of hypertension, symptomatic neuropathy, confirmed coronary artery disease, heart failure or retinopathy were excluded from the study. Patients were selected having at least 3years duration of disease. Written informed consent was obtained from each patient prior to inclusion in the study. Approval from the Institutional Ethics Committee was taken before commencing the study.

\section{Study protocol}

Each subject was given rest for at least 20 minutes before carrying out the actual tests. Standard 12- lead electrocardiogram was taken, and resting heart rate was calculated from recording of ECG lead -II. All five Ewing`s tests were performed as following for the detection of DCAN (diabetic cardiac autonomic neuropathy):

Valsalva Maneuver: By the patient blowing into a mouth piece connected to a sphygmomanometer and holding it at a pressure of 40 $\mathrm{mmHg}$ for 15 seconds while a continuous ECG was recorded. The maneuver was performed 3 times with an interval of one minute in between. The result was expressed as the Valsalva ratio. The mean of three Valsalva ratios was taken as the final value (normal Valsalva ratio 1.21 ; borderline between 1.11 and 1.20 ; abnormal $<1.10$ ).

Heart rate variation during deep breathing: The patient sat quietly and breathed deeply at 6 breaths a minute ( 5 seconds in, and 5 seconds out) for one minute. An ECG was recorded throughout the each inspiration and expiration. The maximum and minimum R-R intervals during each breathing cycle were measured and converted to beats/minute. The result was then expressed as the mean of the difference between maximum and minimum heart rates for the 6 measured cycles in beats/minute; (normal response $>15$ beats/minute, borderline 11-14 beats/minute; abnormal response $<10$ beats/minute).

Immediate heart rate response to standing: The test was performed with the patient lying quietly on a couch while heart rate was recorded continuously on the ECG machine. The patient was asked to stand up unaided and the point at starting to stand was marked on the ECG. The shortest R-R interval at or around the 15th beat and largest R-R interval at or around the 30th beat after starting to stand was measured with a ruler. the characteristic heart rate response was expressed by 30 - 15 ratio (which is normal if $>1.04$; borderline between 1.01 and 1.03; and abnormal if $<1.00$ ).

BP response to standing: The test was performed by measuring the patient's BP while he was lying down quietly and again when he stood up. The fall in BP after 2 minutes was taken as the difference between systolic BP lying and the systolic BP standing (normal response $<10 \mathrm{mmHg}$; borderline 11-29 $\mathrm{mmHg}$; abnormal response $>30 \mathrm{mmHg}$ ).
BP response to sustained handgrip: After instructions in using hand grip of an inflated BP cuff, the subject gripped maximally with his dominant arm for a few seconds; this was repeated thrice. Highest of the 3 readings is called maximum voluntary contraction. Now the subject was instructed to maintain hand grip. The result was expressed as the difference between the highest DBP during hand grip exercise and mean of 3 DBP readings beforehand grip began (normal response $>16$ mmHg; borderline 11-15 mmHg; abnormal < $10 \mathrm{mmHg}$ ).

Results were further analyzed by following criteria:

Normal $=$ all tests normal or 1 test borderline.

Early $=$ one of the three heart rate tests abnormal or two borderline.

Definite $=$ two heart tests abnormal.

Severe $=$ two heart tests abnormal + one or both BP tests abnormal.

Statistical analysis was done using appropriate statistical tests with help of SPSS version 20.0. Statistical significance was considered when $P$ value was $<0.05$. Data was presented as simple mean and standard deviation for quantitative data and as proportions for qualitative data.

\section{Results}

There was a total of 144 cases, out of which 72 were type 2 diabetic patients and healthy controls were 72 , were included in the study group after using proper exclusion criteria.

There was male $(71.5 \%)$ predominating over females $(28.5 \%)$. Mean age of controls (49.69 years) was not significantly different from cases (49.72 years). BMI was normal in both groups still it was significantly higher in cases (24.78) than controls (22.01) (Table 1).

Table I Baseline characteristics of controls and cases

\begin{tabular}{|c|c|c|c|}
\hline \multirow[t]{2}{*}{ Characteristics } & \multicolumn{2}{|c|}{$\begin{array}{l}\text { Study } \\
\text { population }\end{array}$} & \multirow[b]{2}{*}{ P value } \\
\hline & Controls & Cases & \\
\hline Subjects (n) & 72 & 72 & \\
\hline Gender & 69 & 34 & \\
\hline M & 3 & 38 & \\
\hline
\end{tabular}

$103(71.5 \%) 41(28.5 \%)$

$\begin{array}{llcc}\text { Age (Years) } & 49.69 \pm 9.1 & 49.72 \pm 8.8 & >0.05 \\ \text { BMI }\left(\mathrm{Kg} / \mathrm{m}^{2}\right) & 22.01 \pm 4.3 & 24.78 \pm 3.9 & <0.05 \\ & & & \\ \text { 2-h Plasma glucose (m.mol/l) } & 7.1489 \pm 0.38 & 14.90 \pm 4.92 & <0.001^{*} \\ \text { FBS (m.mol/1) } & 4.89 \pm 0.45 & 9.024 \pm 2.9 & <0.001^{*}\end{array}$

All the values are presented as $M E A N \pm 8 . I, P$ value represent the level of significance, *represents statistically significant difference

$40.3 \%$ of the patients had positive CAN whereas $59.7 \%$ had no CAN. Early and definite type of CAN was $13.9 \%$ and $12.5 \%$ respectively whereas severe CAN was present in $13.9 \%$ of cases. Mean duration of diabetes among no CAN group was $8.00+4.37$ and among CAN was 10.9 \pm 7.05 years respectively (Table 2 ).

Numbness and constipation was the most common neuropathic symptom constituting $76 \%$ and $70 \%$ of DCAN cases. Heart rate 
response to deep breathing was the most sensitive test and identified abnormal in $36 \%$ of cases.

Table 2 Comparison of cardiovascular autonomic neuropathy among cases and controls

\begin{tabular}{llll}
\hline Autonomic neuropathy & Controls & Cases & P value \\
\hline Normal & $58(80.6 \%)$ & $43(59.7 \%)$ & \\
Early CAN & $14(19.4 \%)$ & $10(13.9 \%)$ & $<0.001^{*}$ \\
Definite CAN & $0(0 \%)$ & $9(12.5 \%)$ & \\
Severe CAN & $0(0 \%)$ & $10(13.9 \%)$ & \\
\hline Total CAN & $\mathbf{1 4}(\mathbf{1 9 . 4 \% )}$ & $\mathbf{2 9}(\mathbf{4 0 . 3} \%)$ &
\end{tabular}

All the values are presented as No. of subjects and percentage, $P$ value represent the level of significance, *represents statistically significant difference

\section{Discussion}

Cardiovascular autonomic neuropathy (CAN) is a weakness of autonomic control of the cardio vascular system; it reduces the quality of life as well as survival of diabetic patients. Autonomic nerve fibers from both parasympathetic and sympathetic branches become damaged at early stages of CAN. Patients found to have subclinical $\mathrm{CAN}$ at their first-time diagnoses of T2DM or even develop within a year of being diabetic; and two years after diagnosis of type I diabetes but, unfortunately, it remains asymptomatic for several years. ${ }^{10-12}$

In present study CAN was present in 29 patients (40.3\%) out of 72 patients. The prevalence of CAN shifts broadly from 36 to $80 \%$ in India ${ }^{13}$ to $22.2 \%$ in $\mathrm{China}^{14}$ and $25 \%$ in Egypt ${ }^{15}$ etc. Hence, literature revealed a very high prevalence of DCAN in diabetic patients $(30 \%$ to $80 \%$ ), and our results are in accordance with previous studies performed in different countries. In present study that heart rate variability test with deep breathing was found to be very sensitive test in identifying DCAN in asymptomatic patients. However, Ewing`s all five tests are recommended for diagnosis and confirmation of DCAN in patients.

In the present study, age is not found to be a risk factor for development of DCAN however, duration of diabetes was significantly correlated with the severity of CAN. Other studies ${ }^{16,17}$ also reported that mean duration of diabetes was higher in patients with CAN as compare to No CAN patients. Poor glycemic status for prolonged period of time in context of diabetes may represent as vital factor for development and progression of DCAN. Numbness, resting tachycardia and constipation were the most common symptoms.

CAN is one of the most ignored manifestation with respect to diabetes, have higher mortality rate than diabetics without CAN. It is responsible for exercise intolerance, intraoperative liabilities related to heart, hypotension on postural change, silent cardiac ischemia and excessive risk of deaths.

\section{Conclusion}

On the basis of our study results, we recommend a multicenter study to be conducted in all provinces of Pakistan, with larger sample size that would represent the actual status of DCAN in Pakistani type II diabetic population. Also, as the Cardiac autonomic reflex tests (CARTs) as described by Ewing's criteria are simple and reproducible diagnostic tool for evaluation cardiac autonomic neuropathy in diabetic patients, all diabetic patients whether symptomatic or asymptomatic should be routinely evaluated for presence of CAN using CARTs at the time of diagnosis and every five years thereafter.

\section{Acknowledgments}

None.

\section{Conflicts of interest}

The authors declare there is no conflict of interest.

\section{References}

1. International Diabetes Federation (IDF). Diabetes ATLAS. 8th ed. 2017.

2. Gupta P, Verma N, Bhattacharya S, et al. Association of diabetic autonomic neuropathy with red blood cell aldose reductase activity. Can J Diabetes. 2014;38(1):22-25.

3. Patel PM, Shah T, Krish K. The evaluation of cardiac markers in diabetic and non-diabetic patients with myocardial infarction. International Journal of Biomedical and Advance Research. 2014;5(1):7-9.

4. Boulton AJ, Vinik AI, Arezzo JC, et al. Diabetic neuropathies: a statement by the American Diabetes Association. Diabetes Care. 2005; 28(4):956-962.

5. Spallone V, Bellavere F, Scionti L, et al. Recommendations for the use of cardiovascular tests in diagnosing diabetic autonomic neuropathy. Nutr Metab Cardiovasc Dis. 2011;21(1):69-78.

6. Spallone V, Ziegler D, Freeman R, et al. Cardiovascular autonomic neuropathy in diabetes: clinical impact, assessment, diagnosis, and management. Diabetes Metab Res Rev. 2011;27(7):639-653.

7. Maser RE, Mitchell BD, Vinik AI, et al. The association between cardiovascular autonomic neuropathy and mortality in individuals with diabetes a meta-analysis. Diabetes Care. 2003;26(6):1895-1901.

8. Yun JS, Kim JH, Song KH, et al. Cardiovascular autonomic dysfunction predicts severe hypoglycaemia in patients with type 2 diabetes: a 10 year follow-up study. Diabetes Care. 2014;37(1):235-241.

9. Iqbal Hydrie MZ, Shera AS, Fawwad A, et al. Prevalence of metabolic syndrome in urban Pakistan (Karachi): comparison of newly proposed International Diabetes Federation and modified Adult Treatment Panel III criteria. Metab Syndr Relat Disord. 2009;7(2):119-124.

10. Vinik AI, Maser RE, Mitchell BD, et al. Diabetic autonomic neuropathy. Diabetes Care. 2003;26(5):1553-1579.

11. Jawa A, Bokhari R, Jawad A, et al. Diabetic cardiac autonomic neuropathy in well-controlled diabetics within 1 year of diagnosis. Indian J Endocrinol Metab. 2011;15(Suppl 1):S67-S68.

12. Ramavat MR, Ghugare BW, Dinkar MR, et al. Prevalence of cardiac autonomic neuropathy in patients with diabetes. NJIRM. 2012;3:15-19.

13. Khandelwal E, Jaryal AK, Deepak KK. Pattern and prevalence of cardiovascular autonomic neuropathy in diabetics visiting a tertiary care referral centre in India. Indian J Physiol Pharmacol. 2011;55(2):119127.

14. $\mathrm{Xu} \mathrm{W}$, Zhu Y, Yang X, et al. Glycemic variability is an important risk factor for cardiovascular autonomic neuropathy in newly diagnosed type 2 diabetic patients. Int J Cardiol. 2016;215:263-238.

15. Mahdy M, Gadalla F, Khorshid H. Detection of subclinical diabetic autonomic neuropathy by reflex cardiovascular testing. Presented at 18th European Congress of Endocrinology, 2016, Munich, Germany. Endocrine Abstracts 41 EP241. 
16. Valensi P, Paries J, Attali JR, et al. Cardiac autonomic neuropathy in diabetic patients: influence of diabetes duration, obesity, and microangiopathic complications-the French multicentre study. Metabolism. 2003;52(7):815-820.
17. Philips JC, Marchand M, Scheen AJ. Pulse pressure and cardiovascular autonomic neuropathy according to duration of type 1 diabetes. Diabetes Metab Res Rev. 2009;25(5):442-451. 